European

Thyroid Journal
Eur Thyroid J 2016;5:152-163

DOI: $10.1159 / 000447232$
Received: March 14, 2016

Accepted after revision: May 27, 2016

Published online: August 4, 2016

\title{
Impaired Repressor Function in SUMOylation-Defective Thyroid Hormone Receptor Isoforms
}

\author{
Joachim M. Weitzel \\ Institute of Reproductive Biology, Leibniz Institute for Farm Animal Biology (FBN), FBN Dummerstorf, \\ Dummerstorf, Germany
}

\section{Key Words}

Posttranslational modification · SUMO - Gene expression .

Ligand-dependent transcription factor · Transrepression

\begin{abstract}
Background: Many nuclear receptors are modified by posttranslational modifications. Objectives: The transcriptional activity of thyroid hormone receptors (TRs) is modified by the influence of its ligand (thyroid hormones $\mathrm{T}_{3}$ and $\mathrm{T}_{4}$ ), but is also affected by posttranslational modifications. This study focuses on the SUMOylation of TR isoforms and the consequences on transcriptional activity and promoter occupancy. Methods: SUMOylation of TR wild-type as well as isoform-specific point mutations have been studied in vitro. The promoter occupancy of TR (wild-type and double- or triplemutated versions) and transcriptional cofactors have been investigated in chromatin immunoprecipitation (ChIP) and Re-ChIP analysis. Results: TR is modified by SUMO proteins at defined residues: the isoform TRa is mainly modified at lysines 281 and 387, whereas lysines 50 and 443 are major SUMOylation sites of isoform TR $\beta$. Lysine residues K281 (TRa) and K50 (TR $\beta$ ) are isoform-specific SUMOylation sites influencing differing TR domains, whereas K387 (TRa) and K443 (TRB) are orthologous residues. TRs are targets of all three
\end{abstract}

SUMO variants (SUMO-1, -2 , and -3 ). The transcriptional activity of SUMOylation-defective mutants of TR alters gene transcription from positively and negatively regulated $T_{3}$ target genes. Conclusions: The most pronounced effect is an impaired repressor function of SUMOylation-deficient TR in the absence of $T_{3}$. The transcriptional properties of SUMOylation-defective TRs can be at least in part ascribed to altered interaction with transcriptional cofactors such as SRC-1 and NCoR. Thus, these data indicate that posttranslational modification of TR by SUMOylation contribute to the fine tuning of its transcriptional response maintaining effects on cellular and physiological homeostasis.

\footnotetext{
(c) 2016 European Thyroid Association Published by S. Karger AG, Basel
}

\section{Introduction}

Thyroid hormone (TH) has a profound influence on normal development, differentiation, and metabolism. Genomic actions of THs are mainly mediated and regulated by thyroid hormone receptors (TRs); however, THtransmitted effects are also controlled by nongenomic processes as well as by modifying the ligand itself [1-3]. TRs are encoded by two different genes ( $\alpha$ and $\beta$ genes).

\begin{tabular}{|c|c|}
\hline KARGER & $\begin{array}{l}\text { (๑) } 2016 \text { European Thyroid Association } \\
\text { Published by S. Karger AG, Basel }\end{array}$ \\
\hline $\begin{array}{l}\text { E-Mail karger@karger.com } \\
\text { www.karger.com/etj }\end{array}$ & $2235-0640 / 16 / 0053-0152 \$ 39.50 / 0$ \\
\hline
\end{tabular}

Joachim M. Weitzel

Institute of Reproductive Biology

Leibniz Institute for Farm, Animal Biology (FBN), FBN Dummerstorf

Wilhelm-Stahl-Allee 2, DE-18196 Dummerstorf (Germany)

E-Mail weitzel@fbn-dummerstorf.de 
Due to alternative splicing and alternate translational initiation, at least a dozen functional active TR $\alpha$ and TR $\beta$ isoform protein variants are synthesized. The archetypical TR binds to TH response elements which are located in promoter sequences of target genes, but may also be positioned several thousand base pairs up- or downstream of the regulated gene $[1,4]$. TRs belong to a group of transcription factors whose gene regulation function depends on the presence or absence of their particular ligand (i.e. TH). Liganded TRs commonly recruit a large coactivator complex that possesses or attracts enzymatic activities (e.g. coactivator SRC-1), which alter chromatin of target genes in order to generate an open chromatin structure allowing for proper transcription. In the absence of $\mathrm{T}_{3}$ (triiodothyronine), the unliganded TR undergoes conformational changes and recruits a corepressor complex (e.g. corepressor NCoR). Again, this corepressor complex integrates several enzymatic activities which modify chromatin towards a closed structure, resulting in a transcriptional silent state [5]. Unfortunately, mechanisms of negatively $\mathrm{TH}$-regulated genes in the presence of $\mathrm{T}_{3}$ are less clear $[6,7]$. However, the balanced recruitment of transcriptional cofactors might also play an essential role for this $\mathrm{T}_{3}$-dependent gene regulation.

$\mathrm{TH}$ action has also been described to be modified by posttranslational modifications of TR $[3,8]$. In this context, TR $\beta$ has been described to be phosphorylated at Ser142 within the DNA-binding domain (DBD) via the MAP kinase pathway [9]. This particular serine residue is not conserved in TRa. Thus, the functional consequences of phosphorylation of different TR isoforms remain controversial $[10,11]$. Other modifications of TR have also been confirmed to interfere with its transcriptional activity. For example, $\mathrm{T}_{3}$ can trigger increased ubiquitination of $\operatorname{TR} \beta$ within the ligand-binding domain (LBD), causing proteasomal degradation [12]. Besides phosphorylation and ubiquitination, acetylation at three defined sites within the hinge domain of TRa has also been revealed and probably affects DNA binding and transactivation [13].

Strikingly, TRs have recently also been described to be modified by SUMOylation $[14,15]$. Three SUMO (small ubiquitin-like modifier) proteins (SUMO-1, -2, and -3) are contained by mammals. These SUMO proteins comprise approximately 100 amino acids and can be covalently attached to the $\varepsilon$-amino group of the amino acid lysine. The SUMO-modified lysine is often found within a minimal consensus motif $\psi \mathrm{KX}(\mathrm{E} / \mathrm{D})$ ( $\psi$ : hydrophobic amino acid; K: lysine; $\mathrm{X}$ : any amino acid; $\mathrm{E} / \mathrm{D}$ : glutamate or aspartate). SUMO- 1 is characterized as $\sim 50 \%$ identical to SUMO-2/3, whereas SUMO-2 and SUMO-3 are actually almost equal. In contrast to SUMO-1, which is bound as a monomer, SUMO-2 and - 3 are able to form polymers. The SUMO modification and also the mode of covalently labeling to the target protein are similar to the posttranslational modification by ubiquitin: SUMO like ubiquitin is activated by an E1 enzyme (activating enzyme) in an ATP-dependent manner, conjugated by an E2 enzyme [e.g. UBC9 (ubiquitin-conjugating enzyme 9)], and linked to a substrate by the action of a E3 ligase family member $[16,17]$. Posttranslational modification by SUMOylation is not static. In contrast, due to the activity of SUMO-specific proteases, SUMO modification can be detached from the labeled protein without degrading the substrate protein $[16,17]$. SUMOylation has emerged as a significant regulatory mechanism in cell physiology which is associated with modulating a wide variety of cellular processes, such as transcriptional activity, subcellular localization, or interference with binding partners in a target-specific manner. Modification of a target protein by SUMOylation might also interfere with other posttranslational modifications at the same or a closely adjacent site, and therefore might block or at least attenuate other signal transduction pathways [18, 19]. Several transcription factors (e.g. nuclear receptors) and transcriptional cofactors (e.g. coactivators and corepressors) are verified targets of SUMOylation [20-23]. With this study, I demonstrate that SUMOylation of TRa and $\operatorname{TR} \beta$ is capable of fine-tuning its corresponding transcriptional activity. Since I could prove that certain SUMOylation sites are isoform-specific, this study suggests that a TR-SUMOylation might offer a sophisticated regulation mechanism for an isoform-specific regulation of TR $\alpha$ and TR $\beta$ activity.

\section{Material and Methods}

Isolation and Characterization of DNA Sequences

Human TSHa promoter from -802 to +22 in pGL3, rat mGPDH promoter $B$ from -316 to +109 in pGL2, the $5 x U A S-L u c$ reporter, and the expression plasmids of chicken $\mathrm{TR}_{1}$ and human $\mathrm{TR} \beta_{1}$ [both N-terminally fused with hemagglutinin (HA) and Flag epitopes] in pSG5 and pcDNA3 have been described previously $[24,25]$. Expression plasmids of SUMO-1 wild-type in pHH10B, SUMO-1 $\triangle$ GG in $\mathrm{pHH} 10 \mathrm{~B}$, and UBC9 in pcDNA3 are kind gifts from Frauke Melchior (University of Heidelberg, Germany). The expression plasmid of human $\mathrm{TR} \beta_{1}$ is a kind gift from Peter Hofmann (Charité Berlin, Germany). Various Gal4-DBD-TR fusion proteins have been designed by PCR amplification of chicken TR $a$ fragments which were cloned into pcDNA3 (Life Technologies, Darmstadt, Germany). Various GST fusion proteins have been generated by PCR amplification of TR $\alpha$ or TR $\beta$ fragments and 
cloned into pGEX-2T (GE Healthcare, Munich, Germany). Point mutations were introduced using a self-made site-directed mutagenesis system adapted from the QuickChange kit by Stratagene. All clones were confirmed by DNA sequencing. In silico analysis to predict putative SUMOylation sites of TR was performed with SUMOplot (Abgent, San Diego, Calif., USA) and SUMOsp 2.0 [26].

\section{Cell Culture Experiments}

Human hepatocarcinoma HepG2 and human embryonic kidney HEK293 cells were cultured under standard conditions. Transient transfection experiments were conducted using a modified calcium phosphate technique demonstrated previously [24, 25]. For classical transient transfection experiments on $9.6 \mathrm{~cm}^{2}$ dishes (each containing $\sim 7 \times 10^{5}$ cells), the first $2 \mu \mathrm{g}$ of promoter luciferase reporter (pGL3-basic vector) was mixed with $0.2 \mu$ g of chicken $\mathrm{TRa}_{1}$ in pSG5 or human TR $\beta_{1}$ in pcDNA3 and then stimulated with $100 \mathrm{nM} \mathrm{T}_{3}$ (Sigma Aldrich, Taufkirchen, Germany). For mammalian one-hybrid experiments, $2 \mu \mathrm{g}$ of $5 \mathrm{xUAS}$-Luc reporter plasmids were mixed with $0.4 \mu \mathrm{g}$ of the corresponding Gal4-TRa fusion protein plasmid and stimulated with $100 \mathrm{nM} \mathrm{T}_{3}$. Cells were harvested after a 24-hour incubation, and luciferase activity was determined as depicted previously $[24,25]$ and finally normalized to the total protein concentration of the samples (Bradford, Bio$\mathrm{Rad}$ ) or to a normalizing renilla luciferase expression. Luciferase measurements were carried out in duplicate, and each construct was tested in at least three independent transfection experiments with 2-3 culture dishes per experiment.

\section{Immunoprecipitation}

For detection of SUMOylated TR in cell culture, $2 \mu \mathrm{g}$ of FlagHA-tagged chicken $\mathrm{TRa}_{1}$ in pSG5 or Flag-HA-tagged human $\mathrm{TR} \beta_{1}$ in pcDNA3, $2 \mu \mathrm{g}$ of SUMO-1 (wild-type or mutated version) in $\mathrm{PHH} 10 \mathrm{~B}$, and $2 \mu \mathrm{g}$ of UBC9 in pcDNA3 were transfected into $5 \times 10^{6} \mathrm{HepG} 2$ cells. Cells were lysed, incubated with anti-Flag M2 affinity gel (Sigma Aldrich), and immunoprecipitated [27]. After immunoprecipitation, unmodified TRs were detected by Western blot analysis using an anti-HA antibody (No. 6E2; Cell Signaling, Frankfurt am Main, Germany) and SUMO-modified TRs were detected by anti-SUMO-1 antibody (SUMOlink kit, Active Motif). Amounts of immunoprecipitated proteins were quantified using AIDA Image Analyzer v4.15 (Raytest, Straubenhardt, Germany) and expressed as a ratio of modified relative to unmodified protein concentrations.

\section{In vitro SUMOylation Assay}

Bacterially expressed GST-TR $\alpha$ and GST-TR $\beta$ (wild-type and mutant versions) fusion proteins were produced in Escherichia coli as indicated previously. These fusion proteins contain an HA-tag located C-terminally of the GST and N-terminal of the TR protein portion. The total bacterial lysate derived from $100 \mathrm{ml}$ of $E$. coli culture was completely loaded on GST GraviTrap columns (GE Healthcare) and purified according to the instructions of the manufacturer. The expression of correctly sized proteins was monitored by SDS-PAGE. $500 \mathrm{ng}$ of the purified proteins were introduced into the SUMOlink kit (Active Motif, Rixensart, Belgium) according to the instructions of the manufacturer. After incubation for $3 \mathrm{~h}$ at $30^{\circ} \mathrm{C}$, the SUMOylated samples were directly loaded onto an SDS-PAGE and detected by Western blot analysis using an anti-HA antibody (No. 6E2, Cell Signaling).

\section{Chromatin Immunoprecipitation}

Chromatin immunoprecipitation (ChIP) analyses have been performed as previously shown [27]. Constructs for cotransfection are as described above. For immunoprecipitation, antibodies against HA-Tag (No. Y-11, Santa Cruz), Flag-tag (M2 affinity gel, Sigma Aldrich), SRC-1 (No. ab84, abcam), NCoR (No. ab24552, abcam), and nonspecific IgGs (No. I5006, Sigma Aldrich) were used. For the detection of $\mathrm{mGPDH}$ promoter-bound proteins, the PCR primers $\mathrm{mGPDH}$ for $5^{\prime}$-agctggaggttcctgacttcc- $3^{\prime}$ and mGPDH rev $5^{\prime}$-ctttatgtttttggcgtcttc- $3^{\prime}$ were employed by qPCR (icycler, Bio-Rad). For the detection of TSHa promoter-bound proteins, the primers TSH $\alpha$ for $5^{\prime}$-atggtaattacaccaagtaccc- $3^{\prime}$ and TSHa rev $5^{\prime}$-ctttatgtttttggcgtcttc- $3^{\prime}$ were employed by qPCR (i-cycler, Bio-Rad). After the first immunoprecipitation, Re-ChIP analyses were conducted by incubating the samples with $5 \mathrm{mM}$ DTT for 30 $\min$ at $37^{\circ} \mathrm{C}$. Samples were then diluted 1:50 with Re-ChIP buffer (150 mM NaCl, 20 mM Tris-HCl, 2 mM EDTA, 1\% Triton X-100, $\mathrm{pH} 8.1$ ), incubated with the second antibody overnight at $4^{\circ} \mathrm{C}$, and immunoprecipitated as described. ChIP analyses were normalized in a two-step procedure (1) to the input control of the same sample and (2) to a parallel sample treated with nonspecific IgG. Chromatin occupancy of immunoprecipitated samples was determined relative to the occupancy of TR wild-type without $\mathrm{T}_{3}$ stimulation. The amount of immunoprecipitated samples corresponded to $\sim 0.06 \%$ of those from the input controls. Results of at least three independent ChIP experiments, conducted in duplicates, are shown

\section{Statistical Analysis}

Effects were assessed by one-way analysis of variance. Tests (pair-wise comparisons or comparisons vs. control) were performed by the post hoc Newman-Keuls procedure. Values of $\mathrm{p}<$ 0.05 were considered statistically significant. Results are presented as means \pm SD. Tests were performed with the SAS statistical package (SAS Institute, Cary, N.C., USA) and Sigma Stat of Jandel Scientific Software (Erkrath, Germany).

\section{Results}

\section{TR Is a SUMOylated Protein in Cell Culture \\ Experiments}

I transfected Flag-HA-tagged $\mathrm{TR}_{1}$ or Flag-HA-tagged $\mathrm{TR} \beta_{1}$ into HepG2 cells to analyze whether TR might be modified by SUMOylation (fig. 1a, b). In addition, a wildtype SUMO-1 protein or a mutated protein version (SUMO $\Delta \mathrm{GG}$ ) was cotransfected. The SUMO $\Delta \mathrm{GG}$ protein is devoid of two C-terminal glycine residues which prevent conjugating to the lysine residue within the SUMOylation recognition site of target proteins. TR was immunoprecipitated using an antibody directed against a Flag epitope of TR, and Western blot analyses were performed using an anti-HA antibody in order to detect unmodified protein or by using anti-SUMO antibody in order to detect modified versions of TR. Due to the analysis via the Flag- and HA-tags, only preceding cotransfected 
TR proteins are detectable in this assay, whereas no protein could be detected in Western blot analyses without previous cotransfection of a tagged protein version. As shown in figure 1a and $\mathrm{b}$, both TR isoforms are targets of SUMOylation. The amounts of posttranslationally modified TR appears to by higher in the presence of $\mathrm{T}_{3}$; however, this is rather an effect of reduced concentrations of unmodified $\mathrm{TR}$ in the presence of $\mathrm{T}_{3}$. This data indicates that TR is a target of SUMOylation in cell culture experiments.

Further, sequence inspections identified three putative SUMOylation sites in chicken $\mathrm{TRa}_{1}$ at K281, K286, and $\mathrm{K} 387$, all located in the LBD of the protein (fig. 1c). In the human TR $\beta_{1}$ protein, four putative SUMOylation sites were detected. These lysine residues are located within the N-terminal activation domain AF-1 (K50), the hinge domain (K263), and the LBD (K342 and K443) (fig. 1c). Two of the SUMOylation sites positioned in the LBD are conserved between the two TR isoforms (i.e. K286 and K387 of TR $\alpha$ correspond to K342 and K443 of TR $\beta$ ). In contrast, putative SUMOylation sites K281 of TR a and $\mathrm{K} 50 / \mathrm{K} 263$ of TR $\beta$ are unique residues within their particular isoforms (fig. 1c).

\section{SUMOylation of TR $\alpha$ and TR $\beta$ at Defined Residues}

To evaluate the SUMOylation pattern of TRa and $\mathrm{TR} \beta$, I investigated several single, double, and triple mutations in an in vitro SUMOylation assay. In this context, the central lysine residue within the SUMOylation motif was mutated into the amino acid arginine. This mutation is the most conservative mutation, changing one basic amino acid into the second one. However, it has been shown previously that arginine (in contrast to lysine) is not able to be modified by SUMOylation. Furthermore, three single point mutations in the TRa protein were integrated, changing the lysine residues 281, 286, and 387 into arginine (mutants K281R, K286R, and K387R, respectively). Moreover, a double mutant (K281,387R) and a triple mutant $(\mathrm{K} 281,286,387 \mathrm{R})$ of TR $\alpha$ were generated. The wild-type and mutated proteins were introduced to an in vitro SUMOylation assay. Quantification of SUMOylated proteins was performed by comparing the upper band (SUMOylated protein) relative to the lower band (unmodified protein). Again, the in vitro SUMOylation assay not only demonstrated that TRa is a target of SUMOylation (fig. 2) but also that TRa is SUMOylated by all three SUMOylation isoforms: SUMO-1, SUMO-2, and SUMO-3 (fig. 2a-c). Additionally, the major SUMOylation sites appear to be K281 and K387, which were most apparent when SUMO-2/3 mod-

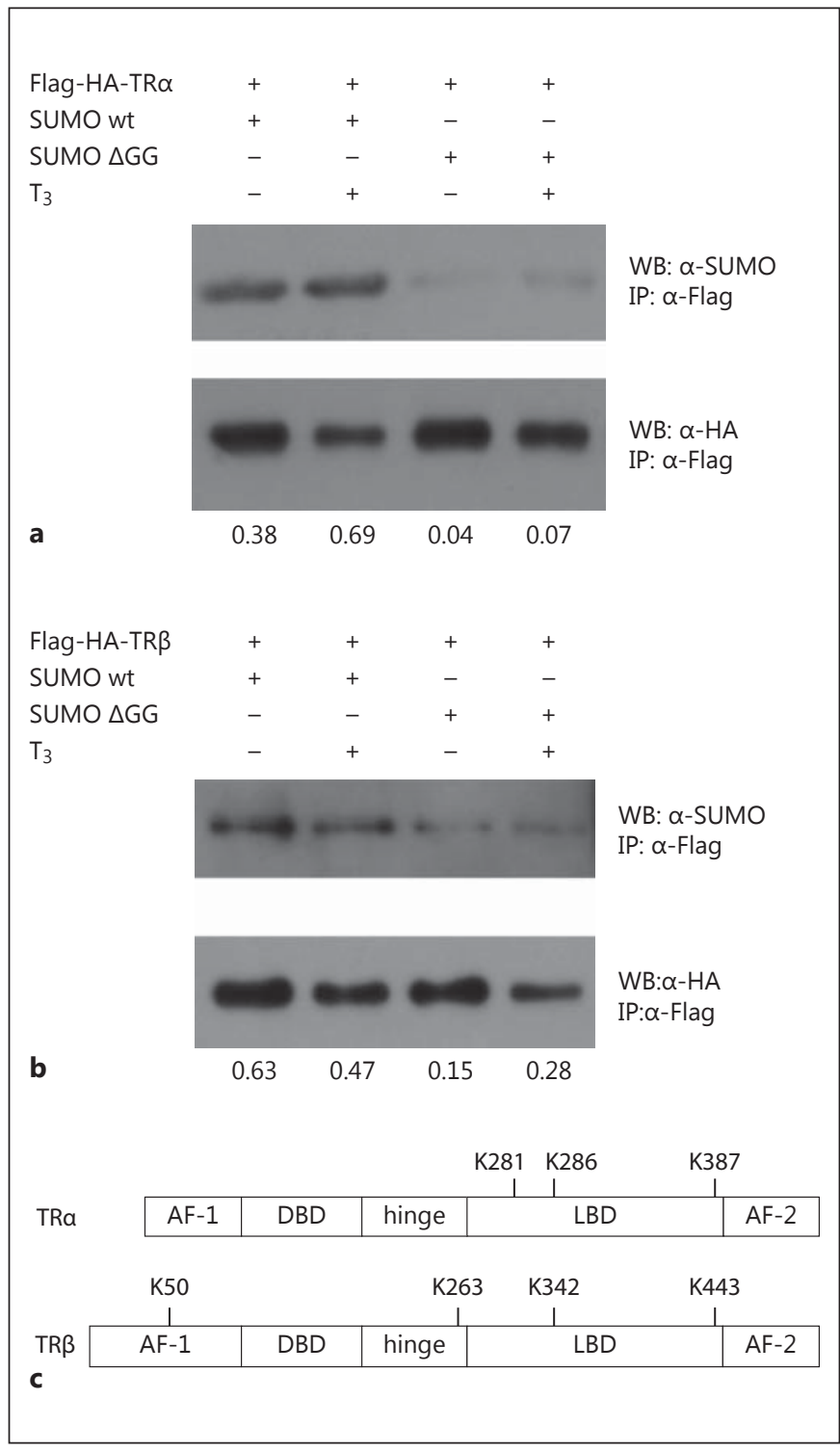

Fig. 1. TR is a SUMOylated protein. Expression plasmids of FlagHA-tagged TR $\alpha$ wild-type (Flag-HA-TR $\alpha$ ) (a) or Flag-HA-tagged TR $\beta$ wild-type (Flag-HA-TR $\beta$ ) (b), SUMO wild-type (SUMO wt), or SUMO $\triangle$ GG were transfected into HepG2 cells and stimulated with or without $\mathrm{T}_{3}$. Cells were immunoprecipitated with an antiFlag antibody ( $\alpha$-Flag) and Western blot analyses were performed using an anti-HA antibody ( $\alpha-\mathrm{HA})$ in order to detect unmodified TR or anti-SUMO antibody ( $\alpha$-SUMO) in order to detect SUMOylated protein versions. Numbers indicate the quantification of the SUMOylated relative to the unmodified protein version. c In silico analysis identified 3 putative SUMOylation sites in chicken TRa (K281, K286 and K387) and 4 putative SUMOylation sites in human TR $\beta$ (K50, K263, K342 and K443). Abbreviations: AF-1 $(-2)=$ activation function-1 $(-2)$; IP = immunoprecipitation; $\mathrm{WB}=$ Western blot. 
ification of TR occurred (fig. 2b, c). However, noncanonic SUMOylation sites might exist since even the triple mutant is a target of SUMOylation in the in vitro assay.

In a similar approach, I established four single point mutations into TR $\beta$, yielding the mutants K50R, $\mathrm{K} 263 \mathrm{R}, \mathrm{K} 342 \mathrm{R}$ and $\mathrm{K} 443 \mathrm{R}$, respectively. The in vitro SUMOylation assay confirmed the SUMOylation of TR $\beta$ by all three SUMOylation isoforms (fig. 2). Further on, the major SUMOylation sites seem to be K50 and K443, and - again - this modification was mostly pronounced by SUMO-2/3 conjugation of TR (fig. 2e, f).

\section{Effect of SUMOylation-Defective Mutants in Cell \\ Culture Experiments}

To test whether SUMOylation-defective mutants might have altered transactivation capacity, I performed classical transient transfection assays on natural promoter constructs upon stimulation by TH. In this assay a reporter construct of a $\mathrm{T}_{3}$ target gene was transfected together with wild-type or mutated variants of TR into HepG2 cells and subsequently stimulated with $T_{3}$.
Without $\mathrm{TR}$ cotransfection, no $\mathrm{T}_{3}$-mediated gene expression was observed (fig. 3). As shown in figure 3, TRa SUMOylation-defective mutants K281 and K387 and TR $\beta$ SUMOylation-defective mutants K50 and K443 show alterations on a negatively $\mathrm{T}_{3}$-regulated TSHa promoter. This observation is in agreement with a previous report on GH and TSH $\beta$ promoters [14].

To further address this issue and to reduce the complexity of the cell system, I conducted mammalian onehybrid assays by investigating the $\mathrm{T}_{3}$-dependent effect of Gal4-DBD-TR-fusion proteins on a UAS-Luc reporter. In a first approach I designed several Gal4-TRa fusion proteins (fig. 4a). Firstly, the full-length $T R \alpha_{1}$ protein (from amino acid 1 to 408) was fused C-terminally to the DBD of Gal4 [construct Gal4-TR(1-408)]. Secondly, for the construct AF1-Gal4-TR(119-408), the endogenous $\mathrm{DBD}$ of TR $\alpha$ was replaced by the Gal4-DBD. Thirdly, the N-terminal activation domain AF-1 and the endogenous DBD of TR was compensated by Gal4-DBD [Gal4-TR(119-408)]. Finally, for the construct Gal4TR(218-408), the AF-1, TR-DBD, and the hinge do-
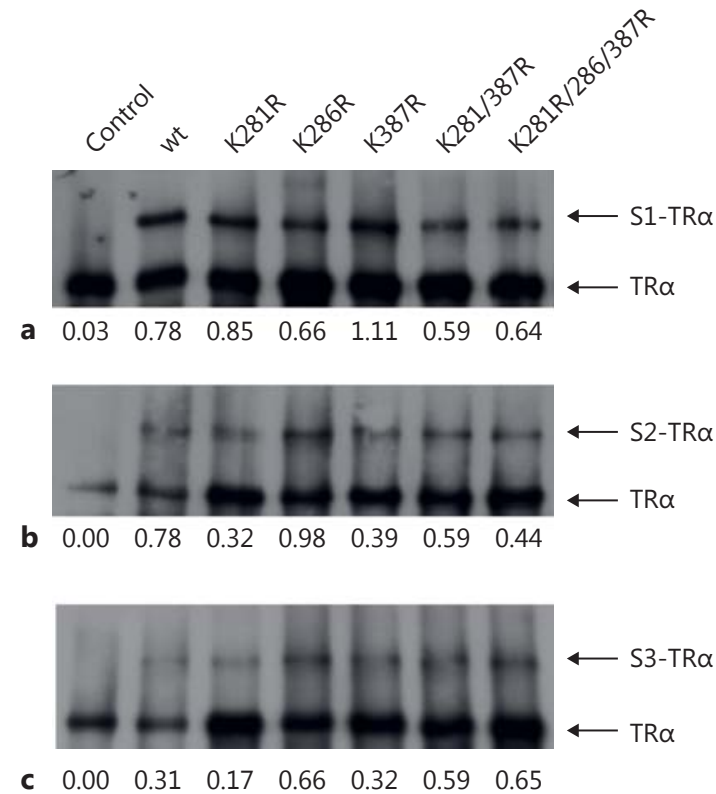

Fig. 2. In vitro SUMOylation of TRa and TR $\beta$. a-c SUMOylation of TR $\alpha$ by SUMO-1 (S1) (a), SUMO-2 (S2) (b), and SUMO-3 (S3) (c) was identified. TRa wild-type (wt), the indicated single point mutants, a double mutant $\mathrm{K} 281 / 387 \mathrm{R}$, and a triple mutant $\mathrm{K} 281 / 286 / 387 \mathrm{R}$ were applied to an in vitro SUMOylation assay. SUMOylated and unmodified TR were detected by Western blot analysis and are indicated by arrows. $\mathbf{d - f}$ SUMOylation of TR $\beta$ by
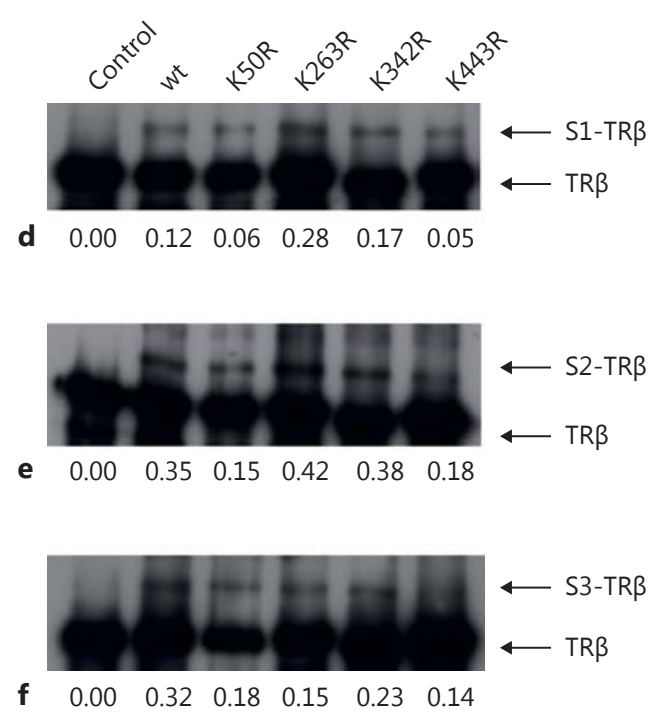

SUMO-1 (S1) (d), SUMO-2 (S2) (e), and SUMO-3 (S3) (f) was also recorded. TR $\beta$ wt and the indicated single point mutants were applied to an in vitro SUMOylation assay and detected by Western blot analysis and quantified. Numbers indicate the quantification of the upper (SUMOylated) relative to the lower (unmodified) band. SUMOylated and unmodified TR are denoted by arrows. 
main were substituted by Gal4-DBD. These four constructs are schematically summarized in figure $4 \mathrm{a}$. As shown in figure $4 \mathrm{~b}$, the constructs Gal4-TR(1-408), AF1-Gal4-TR(119-408), and Gal4-TR(119-408) responded properly to $\mathrm{T}_{3}$ stimulation, whereas Gal4TR(218-408) apparently failed. Hence, I decided to integrate SUMOylation-defective point mutants into the context of the construct Gal4-TR(119-408).
As illustrated in figure 4c, Gal4-TR(119-408) wildtype responded appropriately to $\mathrm{T}_{3}$ stimulation. $\mathrm{T}_{3}$-mediated activation of this construct led to a 12.9-fold induction of reporter gene activity compared to unstimulated expression rates. For the majority of SUMOylation-defective mutants, the activation rates were reduced in comparison to the wild-type Gal4TR(119-408) fusion protein. Intriguingly, the most pro-

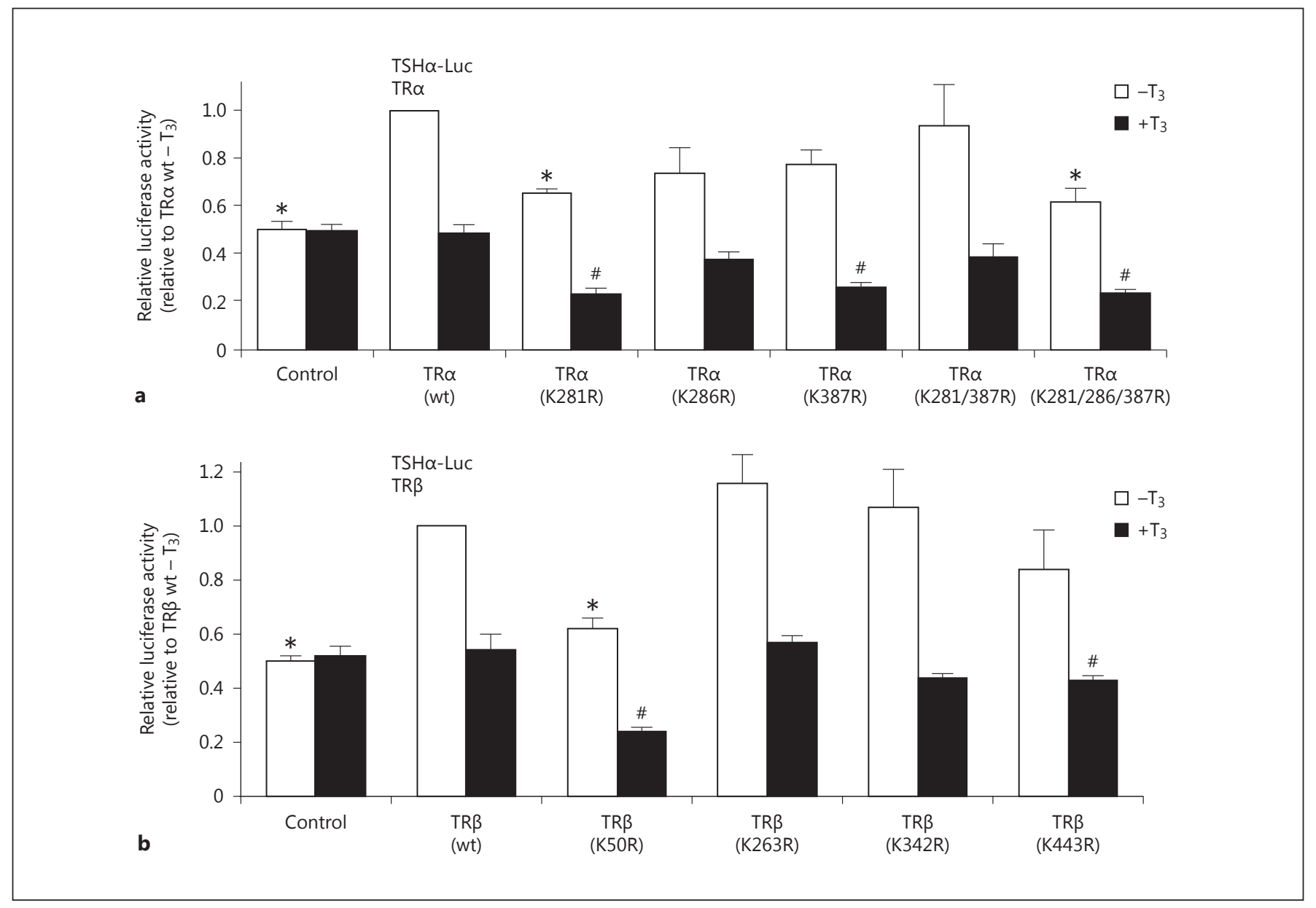

Fig. 3. Regulation of a TSHa promoter by TR isoforms and mutated protein versions in response to $\mathrm{T}_{3}$. A TSH $\alpha$ promoter luciferase reporter was transfected together with TRa wild-type (wt) or the indicated mutants thereof (a), or together with TR $\beta$ wild-type and mutated versions (b) into HepG2 cells and subsequently stim-

Fig. 4. Influence of TR SUMOylation-defective mutants in a mammalian one-hybrid assay. a Schematic overview of different Gal4DBD-TR fusion proteins. Numbers indicate the position of the diverse functional domains within the protein sequence. ${ }^{*} \mathrm{p}<0.05$ relative to unstimulated, corresponding wild-type levels; ${ }^{*} \mathrm{p}<0.05$ relative to stimulated wild-type (wt) levels. b, c Expression vectors ulated with $\mathrm{T}_{3}$. Expression rates are illustrated relative to those of the corresponding wild-type $\mathrm{TR}$ isoform without $\mathrm{T}_{3}$ stimulation \pm SD. ${ }^{*}$ Indicates $\mathrm{p}<0.05$ relative to wild-type and unstimulated and \# indicates $\mathrm{p}<0.05$ relative to wild-type and stimulated levels.

of the indicated Gal4-TR fusion proteins (wild-type or mutated versions) together with an UAS-Luc reporter vector have been transfected into HepG2 cells and stimulated with $\mathrm{T}_{3}$. c The induction rates $\pm T_{3}$ are indicated. Expression rates are displayed in relation to those of the corresponding wild-type Gal4-TR version without $\mathrm{T}_{3}$ stimulation $\pm \mathrm{SD}$.

(For figure see next page.) 
TR $\alpha$ (full length)

\begin{tabular}{|c|c|c|c|c|}
\hline \multicolumn{1}{|c|}{48} & \multicolumn{2}{|c|}{119} & 397 & 408 \\
\hline AF-1 & DBD & hinge & LBD & AF-2 \\
\hline
\end{tabular}

Gal4-TR $\alpha(1-408)$

\begin{tabular}{|c|c|c|c|c|c|}
\hline Gal4-DBD & AF-1 & DBD & hinge & LBD & AF-2 \\
\hline
\end{tabular}

AF1-Gal4-TR $\alpha(119-408)$

\begin{tabular}{|c|c|c|c|c|}
\hline AF-1 & Gal4-DBD & hinge & LBD & AF-2 \\
\hline
\end{tabular}

Gal4-TR $\alpha(119-408)$

\begin{tabular}{|c|c|c|c|} 
Gal4-DBD & hinge & LBD & AF-2 \\
\hline
\end{tabular}

Gal4-TR $\alpha(218-408)$

a

\begin{tabular}{|l|l|l|}
\hline Gal4-DBD & LBD & AF-2 \\
\hline
\end{tabular}
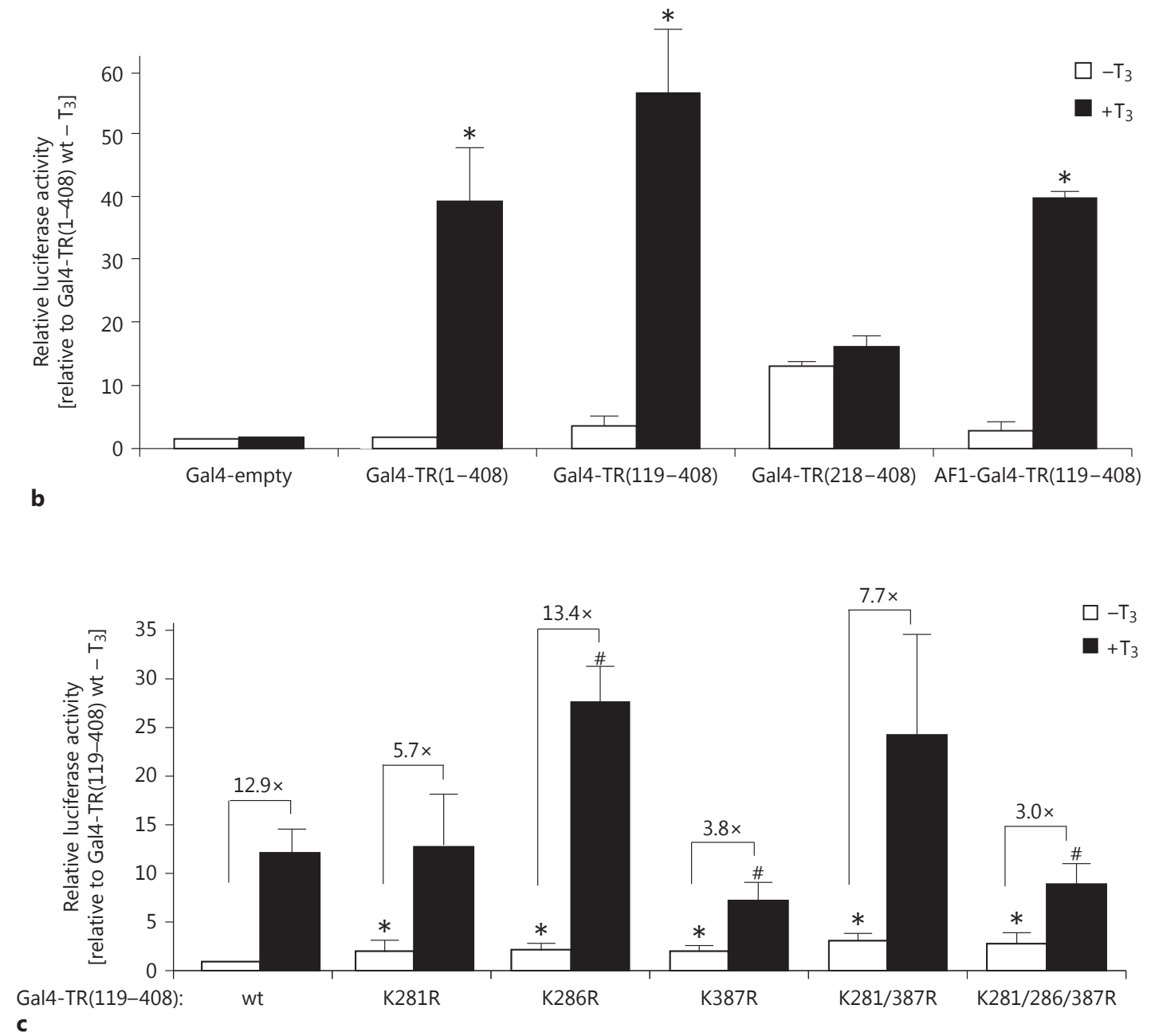

4 


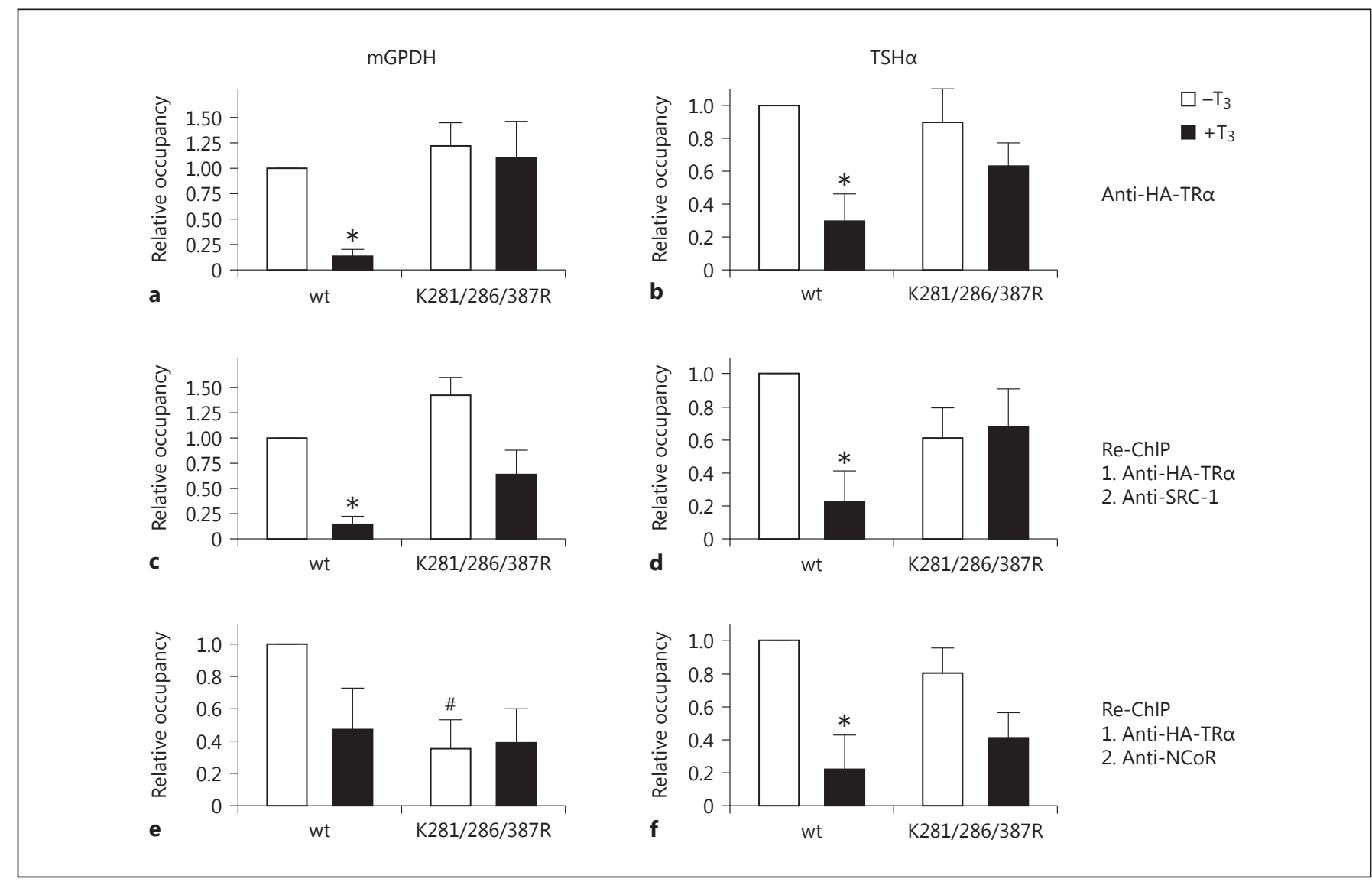

Fig. 5. ChIP and Re-ChIP analysis of TRa on $\mathrm{mGPDH}$ and TSHa gene promoters. mGPDH or TSHa promoter constructs were transfected together with HA-TRa wild-type (wt) or triple mutant HA-TRa(K281/286/387R) into HepG2 cells. a, b Chromatin was immunoprecipitated with an anti-HA antibody and TR-bound DNA was quantified by qPCR. After the first immunoprecipitation

nounced effects were detected for the single mutants K281R (5.7-fold) and K387R (3.8-fold), as well as for the triple mutant K281,286,387R (3.0-fold), whereas the single mutant K286R remained unaffected (fig. 4c). Remarkably, a second distinct effect on the transactivation capacity of the SUMOylation-defective mutants became apparent in this assay: in the absence of ligand $\mathrm{T}_{3}$ the expression rates of SUMOylation-defective mutants are markedly increased compared to wild-type ( $<<0.05)$. This $\mathrm{T}_{3}$-absence-triggered impact could be demonstrated for all investigated single mutants. However, this enhanced transcription activity was most evident in the double as well as triple mutants (fig. 4c). Thus, it has to be hypothesized that SUMOylation-deficient TRs are restrained in their function to suppress $\mathrm{T}_{3}$-dependent gene expression in the absence of $\mathrm{T}_{3}$. This suggests that the antigen-antibody complex was released and a second immunoprecipitation was performed using an anti-SRC-1 antibody (c, d) or an anti-NCoR antibody (e, f). DNA occupancy was calculated relative to the occupancy of the TR wild-type version without $\mathrm{T}_{3}$ stimulation $\pm \mathrm{SD} .{ }^{*} \mathrm{p}<0.05$ relative to unstimulated wild-type levels; ${ }^{*} \mathrm{p}<0.05$ relative to unstimulated wild-type levels.

TR-SUMOylation is significant for repressor activity in the unliganded state.

\section{Occupancy of TR Wild-Type and SUMOylation- \\ Defective Mutants on Natural Promoters}

Since the triple mutant TRa $(\mathrm{K} 281,286,387 \mathrm{R})$ delivered the most striking effects in cell-based transactivation assays (fig. 3a, 4c), I analyzed the occupancy of this mutated protein version relative to the wild-type protein on natural promoters of $\mathrm{T}_{3}$ targets. Promoter occupancy was normalized in a two-step procedure. In a first normalization step the expression levels were normalized to the expression levels of the input controls within the same samples. In a second normalization step the expression levels were normalized to the expression levels detected by using an unspecific IgG from the same starting sample. As shown 


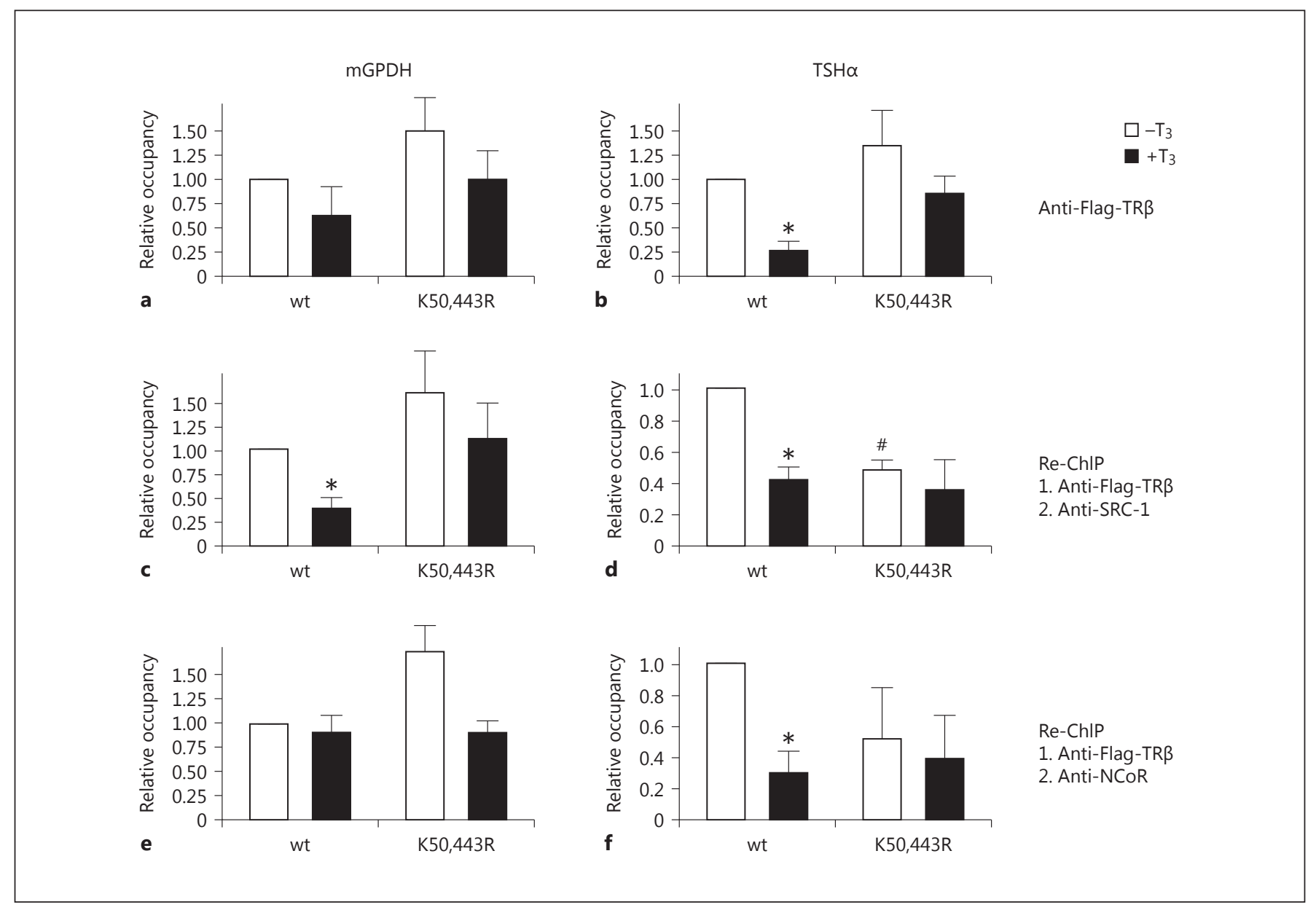

Fig. 6. ChIP and Re-ChIP analysis of TR $\beta$ on $\mathrm{mGPDH}$ and TSHa gene promoters. mGPDH or TSHa promoter constructs were transfected together with Flag-TR $\beta$ wild-type (wt) or double mutant Flag-TR $\beta(K 50,443 R)$ into HepG2 cells. a, b Chromatin was immunoprecipitated with an anti-Flag antibody and TR-bound DNA was quantified by qPCR. After the first immunoprecipitation

in figure 5, a TR wild-type version bound to promoter fragments of $\mathrm{T}_{3}$-regulated target genes in ChIP assays. This holds true not only for a positively regulated $\mathrm{T}_{3}$ target gene promoter (mGPDH, fig. $5 \mathrm{a}, \mathrm{c}, \mathrm{e})$, but also for a negatively regulated one (TSHa, fig. 5b, d, f). Surprisingly, the occupancy of TRa wild-type was reduced in the presence of $\mathrm{T}_{3}$, both on $\mathrm{mGPDH}$ and TSHa genes (fig. $5 \mathrm{a}$, b). On the contrary, this diverse promoter occupancy is completely abrogated by the triple mutant of TRa (fig. 5a, b). In a similar experimental setting, wild-type and the double mutant K50/443R of TR $\beta$ were analyzed (fig. 6).

For the next series of experiments, I conducted Re-ChIP analysis in order to examine the influence of SUMOylation on TR-coactivator-binding (e.g. to SRC-1) and on TR-co- the antigen-antibody complex was released and a second immunoprecipitation was performed using an anti-SRC-1 antibody (c, d) or an anti-NCoR antibody (e, f). DNA occupancy was calculated relative to the occupancy of the TR wild-type version without $\mathrm{T}_{3}$ stimulation $\pm \mathrm{SD} .{ }^{*} \mathrm{p}<0.05$ relative to unstimulated wild-type levels; ${ }^{*} \mathrm{p}<0.05$ relative to unstimulated wild-type levels.

repressor-binding (e.g. to NCoR). To this end, after immunoprecipitation of TR (and release of the first antibody), a second immunoprecipitation was performed by precipitation with an anti-SRC- 1 antibody (fig. 5c, d) or anti-NCoR antibody (fig. 5e, f), respectively. Again, these data unveiled that the promoter occupancy could be altered for the triple mutant relative to the wild-type version of TR. This effect was mostly noticeable for Re-ChIP analysis using an anti-SRC-1 antibody on the negatively regulated TSHa gene (fig. $5 \mathrm{~d}$ ) and an anti-NCoR antibody on the positively regulated $\mathrm{mGPDH}$ gene (fig. 5e). Very similar data have been observed by comparing TR $\beta$ wild-type to the double mutant $\operatorname{TR} \beta(\mathrm{K} 50,443 \mathrm{R})$, indicating identical molecular mechanisms of TR $\alpha$ and TR $\beta$ isoforms (fig. 6). 


\section{Discussion}

THR belong to a family of transcription factors whose transcriptional activity is modulated by the absence or presence of the corresponding ligand, but additionally via various posttranslational modifications. For example, TR $\beta$ has been demonstrated to be phosphorylated at serine-142 within the $\mathrm{DBD}$ [9]. This particular serine residue is not conserved in $\mathrm{TR} \alpha$, thus a specific transcriptional regulation by TR $\beta$ due to phosphorylation appears possible. In line with this argumentation, different DNAbinding and/or transactivation properties of phosphorylated TR $\alpha$ and TR $\beta$ have been described [9-11]. Furthermore, acetylation of TR has been disclosed only for TRa at lysines 128,132, and 134 within the hinge domain [13]. However, since these lysine residues are conserved in the $\beta$-isoform, a similar acetylation of $\operatorname{TR} \beta$ appears likely. Although the lysine residue mentioned above does not reside in classical SUMOylation recognition elements, cross-talk between lysine acetylation and lysine SUMOylation cannot be excluded.

In the present study I described the SUMOylation of $\operatorname{TR} \alpha$ and $\operatorname{TR} \beta$ at defined lysine residues. These findings suggest the major SUMOylation sites of chicken $\mathrm{TR}_{1}$ to be K281 and K387 (corresponds to K283 and K389 of rat $\mathrm{TR}_{1}$ ), both being located in the LBD (fig. 1c). The main SUMOylation sites of TR $\beta_{1}$ appear to be K50 (positioned in the N-terminal activation domain AF-1) and K443 (situated in the LBD) (fig. 2). A preferential SUMOylation of TR $\alpha$ by SUMO-1 and SUMO-2 and preferential SUMOylation of TR $\beta$ by SUMO-3 as described recently [14] could not be observed in the present study (fig. 2). Furthermore, a preferential SUMOylation of TR $\beta$ in the presence of $\mathrm{T}_{3}$ could also not be observed (fig. $1 \mathrm{~b}$ ). Of note, the SUMOylation sites K387 (TR $\alpha)$ and K443 (TR $\beta)$ are known to represent orthologous sequences, thus being conserved in both isoforms (fig. 1c). Hence, targeting this particular site might simultaneously affect both isoforms. Methionine-386 of chicken $\mathrm{TR} \alpha_{1}$ (which corresponds to methionine-388 of rat $\mathrm{TR}_{1}$ ) directly contacts the ligand within the ligand-binding pocket of TRa [28]. Thus, it is tempting to speculate that modification of the immediately adjacent lysine-387 with a bulky SUMO protein will impede the ligand binding of $\mathrm{TR}$ to $\mathrm{T}_{3}$. In contrast to the isoform-conserved lysines, lysine-281 is only detectable in all available TR $\alpha$ variants (e.g. human, mouse, rat, pig, chicken, frog, salmon, Japanese medaka), but in none of the TR $\beta$ proteins. The other way round, lysine-50 is part of the extended AF- 1 domain of TR $\beta$ and therefore not included in TRa. Therefore, specifically targeting K281 in TRa or se- lectively aiming K50 in TR $\beta$ might offer a way to differentially regulate the two TR isoforms. Interestingly, a yeast two-hybrid screen has identified the ubiquitin E3 ligase cullin 1 as a physical interaction partner of TR $\alpha$, but not as a binding partner of TR $\beta$ [29]. Thus, specifically targeting isoform-specific SUMOylation sites might be a way to differentially regulate one particular TR isoform without effecting the SUMOylation (and therefore activity) of the second isoform.

Generally, SUMOylation of proteins is a significant regulator of cell physiology. This posttranslational modification is capable of interfering with multiple cellular processes such as transcriptional activity, subcellular localization, or interference with binding partners. As shown in figures $3 \mathrm{a}$ and $\mathrm{b}$, SUMOylation-defective mutants of TR $\alpha$ (K281 and K387) and TR $\beta$ (K50 and K443) revealed alterations when binding to natural promoters in cell culture experiments. This observation is partially consistent and partially contradictory to recently published data. For example, a TSH $\beta$ reporter construct has been shown to be activated by the mutant TR $\beta$ (K50Q) in the absence of $\mathrm{T}_{3}$ [14]. In contrast, a TSHa reporter construct has been shown to be repressed by the mutant TR $\beta$ (K50R) in the absence of $\mathrm{T}_{3}$ in our experiments (fig. $3 \mathrm{~b}$ ). Since both experiments have been performed using HepG2 cells, differences in the TSH subunit reporter constructs or the investigated mutants (lysine $\rightarrow$ glutamine exchange in the study by Liu et al. [14] versus lysine $\rightarrow$ arginine substitution in this study) might account for some differences. The transactivation capacity of TR did not completely depend on an appropriate posttranslational modification by SUMOylation. Detailed cell-based analyses indicate that SUMOylation-defective TR mutants elicit an altered repressor function in the absence of the ligand (fig. 4c). Specifically, transactivation of the mutant versions is already increased in the absence of the ligand (fig. 4c). Admittedly, the transcriptional activity of these mutants was increased after stimulation with the ligand $\mathrm{T}_{3}$. However, the $\mathrm{x}$-fold $\mathrm{T}_{3}$-mediated activation rates of the SUMOylation-defective mutants K281R and K387R were markedly reduced compared to the expression rates of the wild-type protein version (fig. 4c). These diminished activation rates are mainly attributed to the observed increased transcription activity in the absence of $\mathrm{T}_{3}$. This implies a decreased repressor function of unSUMOylated TR for $\mathrm{T}_{3}$ dependently regulated target genes. This contradicts a previous report which showed a differential SUMOylation in response to ligand binding to the receptor [14]. Additionally, I did not detect an altered subcellular localization of SUMOylation-defective 
mutants (not shown), as this has been described for some SUMOylated nuclear receptors such as AR, PR, or RAR [30-32]. Thus, I have to assume that the primarily effect of SUMOylation incompetence of TR might be an inappropriate repression of gene expression in the absence of $\mathrm{T}_{3}$.

Further on, other results presented above indicate that a SUMOylation of TR within the LBD might interfere with the ligand binding. As the SUMOylation sites K281/ $\mathrm{K} 387$ (TR $\alpha)$ and K443 (TR $\beta)$ are all located within the LBD of TR (fig. 1c), these residues could be attractive candidates for such a regulation mechanism. Additionally, SUMOylation at these sites might interfere with interacting binding partners in a ligand-dependent manner. ChIP analysis underscored this assumption (fig. 5, 6). Concerning promoter occupancy of wild-type and SUMOylation-defective mutant TR, it could be disclosed that the wild-type TR binding on natural promoters, regardless whether on positively or negatively regulated target genes, is reduced following $T_{3}$ delivery (fig. $5 a, b, 6 a$, b). This interesting observation has been noticed in ChIP analyses of TR by several groups [33-36] and might be connected to an altered half-life time depending on the $\mathrm{T}_{3}$ concentration. The differential DNA occupancy of wildtype TR in dependency of $\mathrm{T}_{3}$ is abrogated in the triple mutant $\mathrm{TR} \alpha(\mathrm{K} 281,286,387 \mathrm{R})$ and in the double mutant $\mathrm{TR} \beta(\mathrm{K} 50,443 \mathrm{R})$, underlining the potential significance of posttranslational TR-SUMOylation (fig. 5a, b; 6a, b). ReChIP analyses demonstrated that the TR binding to the coactivator SRC-1 and to the corepressor NCoR is altered in the SUMOylation-defective mutant relative to the wild-type version (fig. $5 c-f, 6 c-f$ ). Consequently, SUMOylation of TR appears to be important for its proper interaction with transcriptional cofactors.

Taken together, the data presented in this paper indicate that TR $\alpha$ and TR $\beta$ are SUMOylated not only at orthologous but also at isoform-specific lysine residues. Furthermore, it could be revealed that SUMOylation of TR in general might interfere with the ligand binding as well as could exert a substantial impact on transcriptional cofactor attachment. Thus, posttranslational modification by SUMOylation contributes to the fine-tuning of transcriptional activity of TR isoforms both on positively as well as on negatively regulated $\mathrm{T}_{3}$ target genes.

\section{Acknowledgements}

The technical assistance of Ursula Antkewitz and Swanhild Rodewald is gratefully acknowledged. I am also indebted to Frauke Melchior and Peter Hofmann for their kind gifts of plasmid DNA, and to Alexander Sobczak for his helpful comments on the manuscript.

\section{Disclosure Statement}

There is no conflict of interest that could be perceived as prejudicing the impartiality of the research reported. This research did not receive any specific grant from any funding agency in the public, commercial or not-for-profit sector. J.M.W. designed the study, performed the research, and wrote the manuscript.

\section{References}

1 Cheng SY, Leonard JL, Davis PJ: Molecular aspects of thyroid hormone actions. Endocr Rev 2010;31:139-170.

$\checkmark 2$ Cioffi F, Senese R, Lanni A, Goglia F: Thyroid hormones and mitochondria: with a brief look at derivatives and analogues. Mol Cell Endocrinol 2013;379:51-61.

-3 Davis PJ, Lin HY, Tang HY, Davis FB, Mousa SA: Adjunctive input to the nuclear thyroid hormone receptor from the cell surface receptor for the hormone. Thyroid 2013;23:15031509.

4 Weitzel JM, Iwen KA: Coordination of mitochondrial biogenesis by thyroid hormone. Mol Cell Endocrinol 2011;342:1-7.

5 Astapova I, Hollenberg AN: The in vivo role of nuclear receptor corepressors in thyroid hormone action. Biochim Biophys Acta 2013; 1830:3876-3881.
6 Weitzel JM: To bind or not to bind - how to down-regulate target genes by liganded thyroid hormone receptor? Thyroid Res 2008;1:4.

7 Santos GM, Fairall L, Schwabe JW: Negative regulation by nuclear receptors: a plethora of mechanisms. Trends Endocrinol Metab 2011; 22:87-93.

8 Pascual A, Aranda A: Thyroid hormone receptors, cell growth and differentiation. Biochim Biophys Acta 2013;1830:3908-3916.

$\checkmark 9$ Lin HY, Zhang S, West BL, Tang HY, Passaretti T, Davis FB, Davis PJ: Identification of the putative MAP kinase docking site in the thyroid hormone receptor-betal DNA-binding domain: functional consequences of mutations at the docking site. Biochemistry 2003; 42:7571-7579.
10 Chen SL, Chang YJ, Wu YH, Lin KH: Mitogen-activated protein kinases potentiate thyroid hormone receptor transcriptional activity by stabilizing its protein. Endocrinology 2003;144:1407-1419.

11 Mochizuki K, Sakaguchi N, Takabe S, Goda T: De-phosphorylation of TRalpha-1 by p44/42 MAPK inhibition enhances T(3)-mediated GLUT5 gene expression in the intestinal cell line Caco- 2 cells. Biochem Biophys Res Commun 2007;359:979-984.

$\checkmark 12$ Dace A, Zhao L, Park KS, Furuno T, Takamura N, Nakanishi M, West BL, Hanover JA, Cheng S: Hormone binding induces rapid proteasome-mediated degradation of thyroid hormone receptors. Proc Natl Acad Sci USA 2000;97:8985-8990. 
13 Sanchez-Pacheco A, Martinez-Iglesias O, Mendez-Pertuz M, Aranda A: Residues K128, 132 , and 134 in the thyroid hormone receptor-alpha are essential for receptor acetylation and activity. Endocrinology 2009;150:51435152.

14 Liu YY, Kogai T, Schultz JJ, Mody K, Brent GA: Thyroid hormone receptor isoform-specific modification by small ubiquitin-like modifier (SUMO) modulates thyroid hormone-dependent gene regulation. J Biol Chem 2012;287:36499-36508.

15 Liu YY, Ayers S, Milanesi A, Teng X, Rabi S, Akiba Y, Brent GA: Thyroid hormone receptor sumoylation is required for preadipocyte differentiation and proliferation. J Biol Chem 2015;290:7402-7415.

16 Meulmeester E, Melchior F: Cell biology: SUMO. Nature 2008;452:709-711.

$\checkmark 17$ Muller S, Hoege C, Pyrowolakis G, Jentsch S: SUMO, ubiquitin's mysterious cousin. Nat Rev Mol Cell Biol 2001;2:202-210.

18 Treuter E, Venteclef N: Transcriptional control of metabolic and inflammatory pathways by nuclear receptor SUMOylation. Biochim Biophys Acta 2011;1812:909-918.

19 Wadosky KM, Willis MS: The story so far: post-translational regulation of peroxisome proliferator-activated receptors by ubiquitination and SUMOylation. Am J Physiol Heart Circ Physiol 2012;302:H515-H526.

20 Chung SS, Ahn BY, Kim M, Kho JH, Jung HS, Park KS: SUMO modification selectively regulates transcriptional activity of peroxisomeproliferator-activated receptor gamma in C2C12 myotubes. Biochem J 2011;433:155161.

21 Rytinki MM, Palvimo JJ: SUMOylation attenuates the function of PGC-1alpha. J Biol Chem 2009;284:26184-26193.
22 Hentschke M, Süsens U, Borgmeyer U: Transcriptional ERRgamma2-mediated activation is regulated by sentrin-specific proteases. Biochem J 2009;419:167-176.

23 Wilson BJ, Tremblay AM, Deblois G, SylvainDrolet G, Giguere V: An acetylation switch modulates the transcriptional activity of estrogen-related receptor alpha. Mol Endocrinol 2010;24:1349-1358.

24 Wulf A, Harneit A, Kroger M, Kebenko M, Wetzel MG, Weitzel JM: $T_{3}$-mediated expression of PGC-1alpha via a far upstream located thyroid hormone response element. Mol Cell Endocrinol 2008;287:90-95.

25 Wulf A, Wetzel MG, Kebenko M, Kroger M, Harneit A, Merz J, Weitzel JM: The role of thyroid hormone receptor DNA binding in negative thyroid hormone-mediated gene transcription. J Mol Endocrinol 2008;41:2534.

26 Ren J, Gao X, Jin C, Zhu M, Wang X, Shaw A, Wen L, Yao X, Xue Y: Systematic study of protein sumoylation: development of a site-specific predictor of SUMOsp 2.0. Proteomics 2009;9:3409-3412.

27 Wulf A, Harneit A, Weitzel JM: $T_{3}$-mediated gene expression is independent of PGC-1alpha. Mol Cell Endocrinol 2007;270:57-63.

28 Wagner RL, Apriletti JW, McGrath ME, West BL, Baxter JD, Fletterick RJ: A structural role for hormone in the thyroid hormone receptor. Nature 1995;378:690-697.

29 Albers M, Kranz H, Kober I, Kaiser C, Klink M, Suckow J, Kern R, Koegl M: Automated yeast two-hybrid screening for nuclear receptor-interacting proteins. Mol Cell Proteomics 2005;4:205-213.
30 Rytinki M, Kaikkonen S, Sutinen P, Paakinaho V, Rahkama V, Palvimo JJ: Dynamic SUMOylation is linked to the activity cycles of androgen receptor in the cell nucleus. Mol Cell Biol 2012;32:4195-4205.

31 Zhu L, Santos NC, Kim KH: Small ubiquitinlike modifier-2 modification of retinoic acid receptor-alpha regulates its subcellular localization and transcriptional activity. Endocrinology 2009;150:5586-5595.

32 Man JH, Li HY, Zhang PJ, Zhou T, He K, Pan $X$, Liang B, Li AL, Zhao J, Gong WL, Jin BF, Xia Q, Yu M, Shen BF, Zhang XM: PIAS3 induction of PRB sumoylation represses PRB transactivation by destabilizing its retention in the nucleus. Nucleic Acids Res 2006;34: 5552-5566.

33 Sharma P, Thakran S, Deng X, Elam MB, Park EA: Nuclear corepressors mediate the repression of phospholipase A2 group IIa gene transcription by thyroid hormone. J Biol Chem 2013;288:16321-16333.

34 Umezawa R, Yamada M, Horiguchi K, Ishii S, Hashimoto K, Okada S, Satoh T, Mori M: Aberrant histone modifications at the thyrotropin-releasing hormone gene in resistance to thyroid hormone: analysis of F455S mutant thyroid hormone receptor. Endocrinology 2009;150:3425-3432.

35 Kim SW, Ho SC, Hong SJ, Kim KM, So EC, Christoffolete M, Harney JW: A novel mechanism of thyroid hormone-dependent negative regulation by thyroid hormone receptor, nuclear receptor corepressor (NCoR), and GAGA-binding factor on the rat $\mathrm{cD} 44$ promoter. J Biol Chem 2005;280:14545-14555.

-36 Mendez-Pertuz M, Sanchez-Pacheco A, Aranda A: The thyroid hormone receptor antagonizes CREB-mediated transcription. EMBO J 2003;22:3102-3112. 